\title{
Safe Human-Robot Interaction in Agriculture
}

\author{
Paul Baxter, Grzegorz Cielniak, Marc Hanheide \\ L-CAS, School of Computer Science \\ University of Lincoln, U.K. \\ \{pbaxter,gcielniak,mhanheide\}@lincoln.ac.uk
}

\begin{abstract}
Robots in agricultural contexts are finding increased numbers of applications with respect to (partial) automation for increased productivity. However, this presents complex technical problems to be overcome, which are magnified when these robots are intended to work side-by-side with human workers. In this contribution we present an exploratory pilot study to characterise interactions between a robot performing an in-field transportation task and human fruit pickers. Partly an effort to inform the development of a fully autonomous system, the emphasis is on involving the key stakeholders (i.e. the pickers themselves) in the process so as to maximise the potential impact of such an application.
\end{abstract}

\section{CCS CONCEPTS}

- Human-centered computing $\rightarrow$ Field studies; • Computing methodologies $\rightarrow$ Cognitive robotics;

\section{KEYWORDS}

Agricultural Robotics; Human-Aware Robot Navigation; Safe HumanRobot Interaction; Side-by-Side Working

\section{ACM Reference Format:}

Paul Baxter, Grzegorz Cielniak, Marc Hanheide and Pál From. 2018. Safe Human-Robot Interaction in Agriculture. In HRI '18 Companion: 2018 ACM/IEEE International Conference on Human-Robot Interaction Companion, March 58, 2018, Chicago, IL, USA. ACM, New York, NY, USA, Article 4, 2 pages. https://doi.org/10.1145/3173386.3177072

\section{INTRODUCTION}

Agricultural environments pose particular problems for the development and deployment of robotics systems, particularly where outdoor environments are concerned. These include the technical challenges imposed by the environment (e.g. weather damage/occlusion of sensors by dirt, the unpredictable form of plants, variable light conditions, etc), and the at present restricted capabilities in terms of automatic robotic grasping. In the presence of co-located and collaborating humans, these issues become even more challenging, as the additional requirements for safety and efficacy need to be considered.

With increased emphasis on automation brought about by changing workforce characteristics and efforts to increase productivity [5], and a current shortfall in technology to manage all processes

Permission to make digital or hard copies of part or all of this work for personal or classroom use is granted without fee provided that copies are not made or distributed for profit or commercial advantage and that copies bear this notice and the full citation on the first page. Copyrights for third-party components of this work must be honored.

For all other uses, contact the owner/author(s).

HRI '18 Companion, March 5-8, 2018, Chicago, IL, USA

(c) 2018 Copyright held by the owner/author(s)

ACM ISBN 978-1-4503-5615-2/18/03.

https://doi.org/10.1145/3173386.3177072

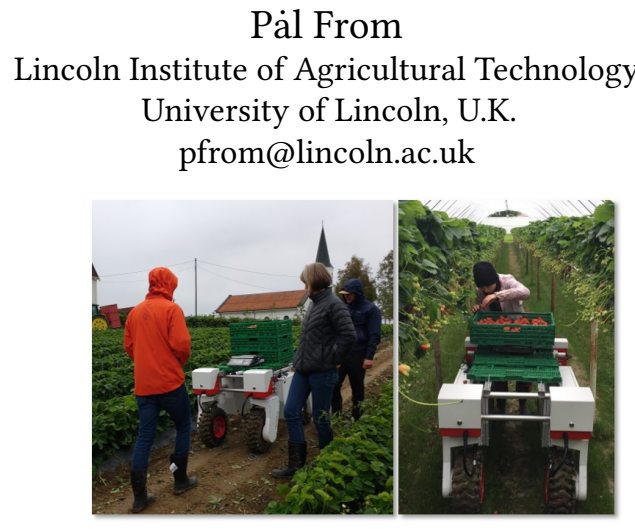

Figure 1: The Thorvald robot in the evaluation environment interacting with pickers: (left) in open strawberry fields, (right) in a polytunnel environment.

automatically, side-by-side working of humans and robots is a current focus of attention. This however requires significant further development [1], with respect to safety and appropriate navigation [3] for example. In this case, the task is to have robots and humans interact in the same space, performing complementary tasks in relation to the same agricultural produce.

It is in this context that we are attempting to develop the technology to facilitate side-by-side working of humans and robots in berry picking scenarios. This is an ideal example for exploration given that at present, human pickers are required to handle the delicate fruit. Our particular focus in this context is the use of robots for in-field transportation: the robots are intended to fetch and carry crates of picked fruit, and deliver these to refrigerated storage units, leaving the pickers to focus on picking. There are a range of challenges to be overcome in attempting to do so beyond the technical challenges outlined above, including human-aware safe navigation, and effective bi-directional communication of intent. Inspired by the principles of user-centred and participatory design, it is also necessary to incorporate the perspective of these workers into the development of the technology, if this is to attain the levels of effectiveness and acceptance desired.

In a wide-scale survey of European countries, agriculture was regarded by the general public as the seventh highest priority area (11\%) for the application of robotics [7]. However, the perspective of people who must/will actually interact with these robots is currently neglected. The aim of this contribution is therefore to lay the foundation of such an effort by characterising their views such that they could be incorporated in the design process of the eventually autonomous robots, with a particular emphasis on perceived safety and acceptance.

\section{PILOT STUDY}

The purpose of the pilot study was to work with the expert human pickers to evaluate how an autonomous robot-based in-field transportation system would work for them, what constraints are 


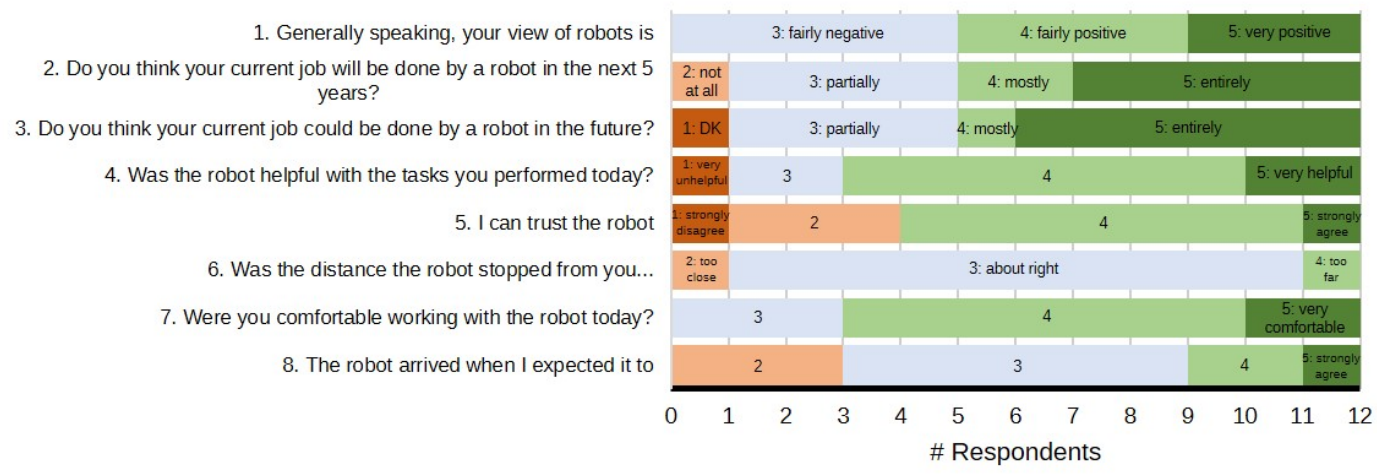

Figure 2: Outcome of questions asked to participants: all were five-point scales, with extrema defined as shown (DK: don't know).

imposed by such a solution, but also what benefits this may confer. In the spirit of participatory design [6], the intention of this study is to fundamentally involve these key stakeholders in the development of the robot system, to characterise their responses to the robot and its role, and to gather data on the appropriateness of the controlled robot behaviour in order to inform the design of the autonomous system.

The study involved 12 experienced subjects (the size of a typical 'gang' of pickers) who were employed for normal strawberry picking activities on a Norwegian farm. These participants were from Poland (7) and Vietnam (5). Over a period of two days, their normal picking activities were augmented by the presence of a Thorvald robot, which acted as their in-field transportation for the picked berries, in both open fields and in polytunnels (figure 1). The robot was remote controlled by a co-located operator: their task was to navigate the robots to the pickers' location when they requested it, allow the filled crates to be loaded onto the robot, and then transport these to the storage facility.

The participants were briefed prior to their interaction with the robot. The intention was to keep their normal working process as intact as possible, replacing only their transport of crates with the robot. Each participant was provided with a wireless button that enabled them to call for a robot when crate pick-up was required. At the end of the working day, the participants were provided with a brief questionnaire (administered in English, but with translation assistance where necessary), and an informal verbal debriefing.

While only an initial small pilot study, the results (figure 2) indicate that after the experience with the robots, they were generally viewed positively (questions 4 and 7), with the behaviour viewed as appropriate (4 and 8 ) and safe (5, 6 and 7). This was despite the participants having mixed views on robots in general (question 1), although they seemed less worried about the prospect of their work being directly replaced by robots ( 2 and 3 ). In contrast to an overall relatively negative view of robots in terms of job replacement [7], it seems that our participant pool have a somewhat more positive perspective despite the direct impact such technology is likely to have. In addition to these results, data on robot behaviour on approaching the participants, and the views of the robot teleoperators were collected, as these will directly inform the design of the autonomous system, as they pertain to perceived safety aspects.

\section{OUTLOOK}

In terms of technical challenges, human-aware robot navigation planning has received significant attention [1], with various techniques employed to take into account, for example, interaction potential [4] or movement prediction [2], which are relevant to this agriculture domain. However, these techniques are generally developed in indoor spaces, and contexts where the environment (including humans) can be reliably detected. Collecting information from 'in field' deployments such as this enable us to address such concerns. In side-by-side working environments, such physical behaviours are clearly of relevance, however, it is also the interaction between the robot and human pickers that is of importance, both in terms of productivity and safety. This pilot study has laid the foundation of an effort to incorporate the views of the target user group in the design process of the autonomous robots.

\section{ACKNOWLEDGMENTS}

This work was supported by the internally funded RASberry project (https://rasberryproject.com/), and partially by the EU H2020 project 732773 ILIAD.

\section{REFERENCES}

[1] Avital Bechar and Clément Vigneault. 2016. Agricultural robots for field operations: Concepts and components. Biosystems Engineering 149 (2016), 94-111. https: //doi.org/10.1016/j.biosystemseng.2016.06.014

[2] Christian Dondrup and Marc Hanheide. 2016. Qualitative Constraints for Humanaware Robot Navigation using Velocity Costmaps. IEEE International Symposium on Robot and Human Interactive Communication (RO-MAN) (2016), 586-592. https: //doi.org/10.1109/ROMAN.2016.7745177

[3] Thibault Kruse, Amit Kumar Pandey, Rachid Alami, and Alexandra Kirsch. 2013. Human-aware robot navigation: A survey. Robotics and Autonomous Systems 61, 12 (2013), 1726-1743. https://doi.org/10.1016/j.robot.2013.05.007

[4] Ross Mead and Maja J. Matarić. 2016. Autonomous human-robot proxemics: socially aware navigation based on interaction potential. Autonomous Robots (2016), 1-13. https://doi.org/10.1007/s10514-016-9572-2

[5] The National Farming Union. 2017. Vision for the future of farming: access to a competent and flexible workforce. Technical Report. National Farming Union, Stoneleigh Warwickshire. https://www.nfuonline.com/assets/97783

[6] Selma Šabanović. 2010. Robots in society, society in robots. International fournal of Social Robotics 2, 4 (2010), 439-450.

[7] TNS Opinion \& Social. 2012. Public Attitudes towards Robots. Technical Report Special Eurobarometer 382. Directectorate-General for Information Society and Media, European Commission. http://ec.europa.eu/digital-agenda/en/news/ dataset-eurobarometer-survey-public-attitudes-towards-robots 